\title{
Research on Soft Measurement of Electronic Stability Control for Electric-wheel Vehicle
}

\author{
Cheng Wang \\ College of Automotive Engineering \\ College of Plant Science \\ Jilin University \\ Changchun, China
}

\author{
Chuanxue Song * \\ College of Automotive Engineering \\ Jilin University \\ Changchun, China \\ * Corresponding Author
}

\begin{abstract}
In order to enhance the timeliness and accuracy of the soft measurement method of the ESC for electricwheel vehicle, an improved soft measurement algorithm was designed. The improved algorithm was built based on an improved UKF. The improved UKF sampling strategy used scaled minimal skew sampling method to speed up the system sampling rate. The sigma points of the improved UKF were sampling two times to enhance the accuracy. The three degree of freedom vehicle model was built to establish vehicle dynamic relationship. According to the three degree of freedom vehicle model, the vehicle measurement model was built. The verification experiments of improved soft measurement algorithm were made based on the electricwheel vehicle ESC test platform. The experiments included a step input experiment in $\mathbf{7 0}$ kilometers per hour and a step input experiment in 110 kilometers per hour. From the experimental results, it could be seen that the estimated value of yaw velocity and sideslip angle tracked the actual very well. The overshoot of the tracking process was small and the lag time was short. The improved soft measurement algorithm was fit for the $\mathrm{ESC}$ of electric-wheel vehicle.
\end{abstract}

Keywords-soft measurement; vehicle model; electricwheel vehicle; improved $\mathrm{UKF}$; ESC

\section{INTRODUCTION}

Electric-wheel vehicle is gradually valued due to its environmental protection and flexible control. The four wheels of electric-wheel vehicle can be controlled independently because a motor is installed in the hub of the wheel [1]. The vehicle ECU (Electronic Control Unit) sends out the control commands to the motor for driving and braking. ESC (Electronic Stability Control) is benefit to the running stability of vehic les. A lot of research works have been done on ESC of the fuel vehicles. But the ESC used by the ordinary vehicles can not be fit for electricwheel vehicle very well. The research areas of ESC for electric-wheel vehicle main include ABS (Antilock Brake System) control, TCS (Traction Control System) control, EBD (Electronic Brake-force Distribution) and AYC (Active Yaw Control). The measurement of vehicle running state parameters is very important for the normal operation of ESC. Some running state parameters can be directly measured by vehicle sensors. Soft measurement technology is widely used in automobile enterprises because the high cost of the vehicles using the sensors to measure the data. The tracking control of yaw velocity and sides lip angle is one of the core issues for ESC. The yaw velocity is usually measured with a hardware sensor. The sideslip angle is usually measured with soft measurement technology [2]. Soft measurement method is needed for these two parameters in ESC bench test.

\section{ESC MEASUREMENT THEORY}

ESC measurement flow is shown as the Fig. 1. The driver system includes steering wheel, accelerator pedal and brake pedal. The steering wheel angle, the distance and speed of the accelerator pedal and the brake pedal are transmitted to the ECU of electric-wheel vehicle to steer, accelerate or brake. The road system provides road adhesion information for ECU to judge road conditions. ECU is the brain of electric-wheel vehicle to control all works. ECU creates vehicle expected running status according to the driver's manipulation intention. Hardware sensors measure vehicle actual running status. At the same time, the estimator relying on soft sensor technology estimates and measures other vehicle actual running status. The errors of the actual running status and the expected running status are the controlled values in ESC [3].

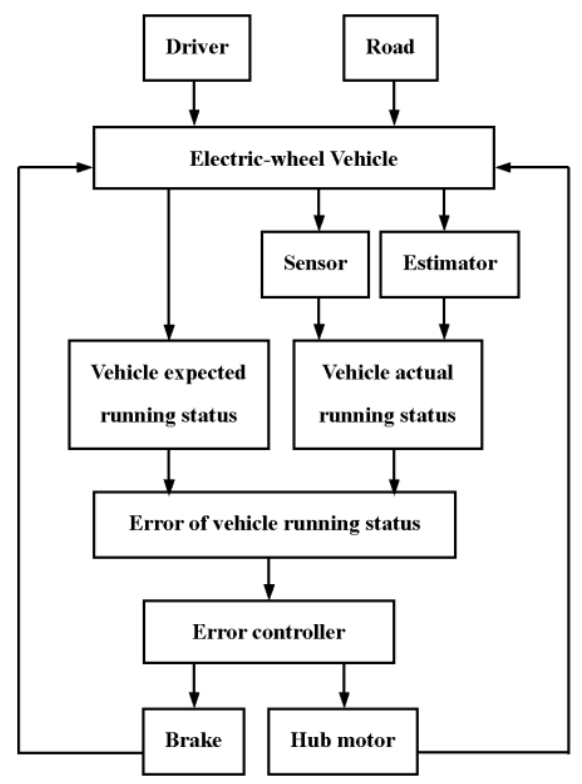

Figure 1. ESC measurement flow block diagram

The running controller controls the error of vehicle running status through the control algorithm. The aim of the running controller is reducing or eliminating the error. 
The controller controls the brake system and the hub motor to change the moment worked on the wheels. The information of the brake system and the hub motor are feed backed to the ECU of electric-wheel vehicle. The estimation theory algorithm is adopted by the estimator to soft measure the actual values.

\section{VEHIClE DyNAMICS MATHEMATICAL MODELING}

\section{A. Three Degree of Freedom Vehicle Model}

Simple and reasonable vehicle mathematical model is an efficient method for the estimation algorith $\mathrm{m}$. The three degree of freedom vehicle model is shown as the Fig. 2. This vehicle model includes vehicle lateral movement, longitudinal movement and yaw movement [4].

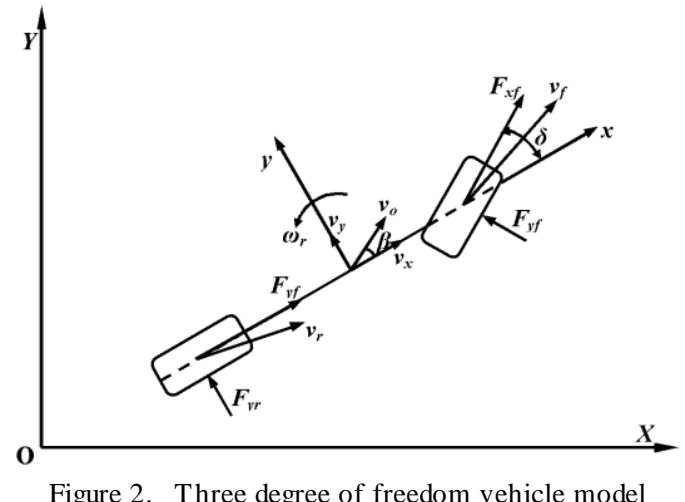

Vehicle longitudinal movement equation is shown as the following equation:

$$
\dot{v}_{x}=a_{x}+v_{x} \omega_{r} \beta
$$

$v_{x}$-longitudinal speed; $a_{x}$-longitudinal acceleration; $\omega_{r}$-yaw velocity; $\beta$-sideslip angle

Vehicle lateral motion equation is shown as the following equation:

$$
\begin{gathered}
m\left(\dot{\beta}+\omega_{r}\right) v_{x}=\left(k_{f}+k_{r}\right) \beta+\frac{\omega_{r}}{v_{x}}\left(l_{f} k_{f}-l_{r} k_{r}\right)-k_{f} \delta_{f} . \\
a_{y}=\frac{1}{m}\left(k_{f}+k_{r}\right) \beta+\frac{\left(l_{f} k_{f}-l_{r} k_{r}\right)}{m v_{x}} \omega_{r}-\frac{k_{f}}{m} \delta_{f}
\end{gathered}
$$

$k_{f}$-front wheel lateral stiffness; $k_{r}$-rear wheel lateral stiffness; $\delta_{f}$-front wheel angle; $m$-vehicle mass; $l_{f^{-}}$distance from centre of mass to the front axle; $l_{r}$-distance from centre of mass to the rear axle.

Vehicle yaw motion equation is shown as the following equation:

$$
J_{z} \dot{\omega}_{r}=\left(l_{f} k_{f}-l_{r} k_{r}\right) \beta+\frac{\omega_{r}}{v_{x}}\left(l_{f}^{2} k_{f}+l_{r}^{2} k_{r}\right)-l_{f} k_{f} \delta_{f}+M_{a} .
$$

$J_{z}$-moment of inertia around $\mathrm{z}$ axis; $v_{y}$-lateral speed; $M_{a^{-}}$ active yaw moment.

The influence coefficients of yaw velocity, sideslip angle and front wheel angle are added to active yaw moment. Active yaw moment equation is shown as the following equation:

$$
M_{a}=k_{\gamma} \omega_{r}+k_{\beta} \beta+k_{\delta} \delta_{f} .
$$

$k_{\gamma}$-yaw velocity influence coefficient; $k_{\beta^{-}}$sideslip angle influence coefficient.

Vehicle yaw motion equation considering the influence coefficient is shown as the following equation:

$$
\begin{aligned}
J_{z} \dot{\omega}_{r}= & \left(l_{f} k_{f}-l_{r} k_{r}+k_{\beta}\right) \beta+\left(\frac{l_{f}^{2} k_{f}+l_{r}^{2} k_{r}}{v_{x}}+k_{\gamma}\right) \omega_{r} . \\
& -\left(k_{\delta}-l_{f} k_{f}\right) \delta_{f}
\end{aligned}
$$

\section{B. Vehicle Soft Measurement Model}

According to the three degree of freedom vehicle model the vehicle state and measurement equations are shown as the following equations [5]:

$$
\begin{gathered}
\left\{\begin{array}{l}
\dot{x}(t)=A x(t)+B u(t)+C w(t) \\
z(t)=D x(t)+E u(t)+F v(t)
\end{array} .\right. \\
\left\{\begin{array}{l}
x=\left[\omega_{r}, \beta\right] \\
u=[\delta] \\
z=\left[a_{y}\right]
\end{array}\right.
\end{gathered}
$$

$$
\left\{\begin{array}{l}
A=\left(\begin{array}{ll}
\frac{l_{f}^{2} k_{f}+l_{r}^{2} k_{r}+k_{\gamma} v_{x}}{J_{z} v_{x}} & \frac{l_{f} k_{f}-l_{r} k_{r}+k_{\beta}}{J_{z}} \\
\frac{l_{f} k_{f}-l_{r} k_{r}-m v_{x}}{m v_{x}^{2}} & \frac{k_{f}+k_{r}}{m v_{x}}
\end{array}\right) ; C=\left(\begin{array}{c}
\frac{1}{m v_{x}} \frac{1}{m v_{x}} \\
\frac{l_{f}}{J_{z}} \frac{l_{r}}{J_{z}}
\end{array}\right) ; D=\left(\begin{array}{c}
\frac{l_{f} k_{f}-l_{r} k_{r}}{m v_{x}} \\
-\frac{m v_{x}}{k_{z}}
\end{array}\right) . \\
E=\left(\begin{array}{c}
0 \\
k_{f}
\end{array}\right) ; F=\left(\begin{array}{c}
\frac{1}{m} \\
\frac{1}{m}
\end{array}\right)
\end{array}\right.
$$

$x$-state variable; $u$-controlled variable; $z$-output variable; $w$ systemnoise; $v$-measurement noise.

\section{IMPROVED ESC SOFT MEASUREMENT ALGORITHM}

The soft measurement algorithm of ESC is state estimation algorithm at present. EKF (Extended Kalman Filter) algorith $m$ is the most applied. EKF is a widely used method of least square error estimation algorithm. EKF is the Taylor expansion of the nonlinear system function at 
the best estimate point. The nonlinear model is simplified as a linear model after the high order is given up. The classical KF (Kalman Filter) is used to complete the filter work after those works. EKF linearization of nonlinear system needs the transfer function of the system can be approximated by a linear function. EKF needs to be carried out by the complex Jacobi matrix calculation meanwhile. These shortcomings make the EKF in the vehicle soft measurement process has limitations [6].

In order to overcome the problem of EKF, UKF (Unscented Kalman Filter) algorithm is generated based on UT (Unscented Transformation). The biggest advantage of $\mathrm{UKF}$ is that it does not need to calculate the Jacobi matrix. UKF is the probability density distribution function of the nonlinear function. The statistical accuracy of the nonlinear function of UKF is at least second order. When UKF is used in the non stationary working environment of vehicles, the UKF must have good adaptive ability and strong robustness. These reasons make it necessary to improve the existing UKF algorithm [7].

\section{A. Initialization Part of Improved UKF}

The scaled minimal skew sampling method is used as the improved UKF sampling strategy. The semi positive stereotype of the prediction covariance is ensured by introducing parameters in the weight. In the ESC system with high real-time requirements, the number of sigma sampling points is $n+2$. The weights equations are shown as the following equations [8]:

$$
\begin{gathered}
\left\{\begin{array}{l}
0 \leq w_{0}<1 \\
w_{1}=\frac{1-w_{0}}{2^{n}} .
\end{array}\right. \\
w_{i}^{m}= \begin{cases}\frac{\left(\varsigma^{2}-1\right) w_{0}}{\varsigma^{4}} & i=0 \\
\frac{1-w_{0}}{2^{n} \varsigma^{2}} & i=1,2 \\
\frac{2^{i-1} w_{1}}{\varsigma^{2}} \quad & i=3,4 \ldots n+1\end{cases} \\
w_{i}^{c}=\left\{\begin{array}{ll}
w_{0}^{m}+\left(1+\xi-\varsigma^{2}\right) & i=0 \\
w_{1}^{m} & i \neq 0
\end{array} .\right.
\end{gathered}
$$

$w_{i}^{m}$-mean value of variance; $w_{i}^{c}$-weighted value of variance

When the number of dimension is one dimension, the initialization process are shown as the following equations:

$$
\left\{\begin{array}{l}
\chi_{0}^{1}=[0] \\
\chi_{1}^{1}=\left[-\frac{1}{\sqrt{2 w_{1}}}\right] . \\
\chi_{2}^{1}=\left[\frac{1}{\sqrt{2 w_{1}}}\right]
\end{array}\right.
$$

When the number of dimension is multi-dimensional, the initialization process is shown as the following equations:

$$
\begin{aligned}
& \left\{\begin{aligned}
& \chi_{i}^{j+1}=\left[\begin{array}{c}
\chi_{0}^{j} \\
0
\end{array}\right] i=0 \\
& \chi_{i}^{j+1}=\left[-\frac{1}{\sqrt{2 w_{j+1}^{m}}}\right] \quad i=1, \ldots j \\
& \chi_{i}^{j+1}=\left[\frac{1}{\sqrt{2 w_{j+1}^{m}}}\right] \quad i=j+1
\end{aligned}\right. \\
& P_{i, 0}^{j}=E\left[\left(x_{i, 0}^{j}-\hat{x}_{i, 0}^{j}\right)\left(x_{i, 0}^{j}-\hat{x}_{i, 0}^{j}\right)^{T}\right] .
\end{aligned}
$$

\section{B. Forecast and Estimation Parts of Improved UKF}

The mean and variance of the predicted sigma points is shown as the following equations [9]:

$$
\begin{gathered}
\chi_{i, k \mid k-1}^{j}=f\left(\chi_{i, k-1}^{j}, u_{i, k-1}^{j}, w_{i, k-1}^{j}\right) . \\
\hat{x}_{i, k \mid k-1}^{j}=\sum_{i=0}^{2 n} w_{i}^{m} \chi_{i,\left.k\right|_{k-1} ^{j}}^{j} . \\
P_{i,\left.k\right|_{k-1} ^{j}}^{j}=\sum_{i=0}^{2 n} w_{i}^{c}\left(\chi_{i, k \mid k-1}^{j}-\hat{x}_{i, k \mid k-1}^{j}\right) \\
\left(\chi_{i, k \mid k-1}^{j}-\left.\hat{x}_{i, k}^{j}\right|_{k-1}\right)^{T}+Q_{i, k}^{j}
\end{gathered} .
$$

The new predicted sigma points are constructed as the following equation:

$$
\left\{\begin{array}{rl}
\tilde{\chi}_{i}^{j+1}=\left[\begin{array}{c}
\hat{x}_{0}^{j} \\
0
\end{array}\right] \quad & i=0 \\
\tilde{\chi}_{i}^{j+1}=\left[\begin{array}{c}
\hat{\mathrm{x}}_{i}^{j} \\
\left.-\frac{1}{\sqrt{2 w_{j+1}^{m}}}\right]
\end{array}\right] \quad i=1, \ldots j \\
\tilde{\chi}_{i}^{j+1}=\left[\frac{1}{\sqrt{2 w_{j+1}^{m}}}\right] \quad i=j+1
\end{array} .\right.
$$

The predicted values of the observed values and observed values are shown as the following equations:

$$
\begin{gathered}
z_{i, k}^{j}=h\left(\left.\tilde{\chi}_{i, k}^{j}\right|_{k-1}, u_{i, k-1}^{j}, v_{i, k-1}^{j}\right) . \\
\hat{z}_{i, k}^{j}=\sum_{i=0}^{2 n} z_{i, k}^{j} .
\end{gathered}
$$


The update of the state mean and variance are shown as the following equations [10]:

$$
\begin{gathered}
\hat{x}_{i, k}^{j}=\hat{\chi}_{i, k \mid k-1}^{j}+k_{i, k}\left(z_{i, k}^{j}-\hat{z}_{i, k}^{j}\right) . \\
P_{i, k}^{j}=P_{i, k \mid k-1}^{j}-k_{i, k} P_{z i, k}^{j} k_{i, k}^{T} . \\
k_{i, k}=P_{x_{i, k} z_{i, k}}^{j}\left(P_{z i, k}^{j}\right)^{-1} . \\
P_{x_{i, k} z_{i, k}}^{j}=\sum_{i=0}^{2 n} w_{i}^{c}\left(\chi_{i, k \mid k-1}^{j}-\left.\hat{x}_{i, k}^{j}\right|_{k-1}\right)\left(z_{i, k}^{j}-\hat{z}_{i, k}^{j}\right) . \\
P_{z i, k}^{j}=\sum_{i=0}^{2 n} w_{i}^{c}\left(z_{i, k}^{j}-\hat{z}_{i, k}^{j}\right)\left(z_{i, k}^{j}-\hat{z}_{i, k}^{j}\right)^{T}+R_{i, k}^{j} .
\end{gathered}
$$

\section{EXPERIMENT}

The verification experiments of improved soft measurement algorithm were done based on the electricwheel vehicle ESC test platform. The main structure of the test platform is shown as the Fig. 3 .

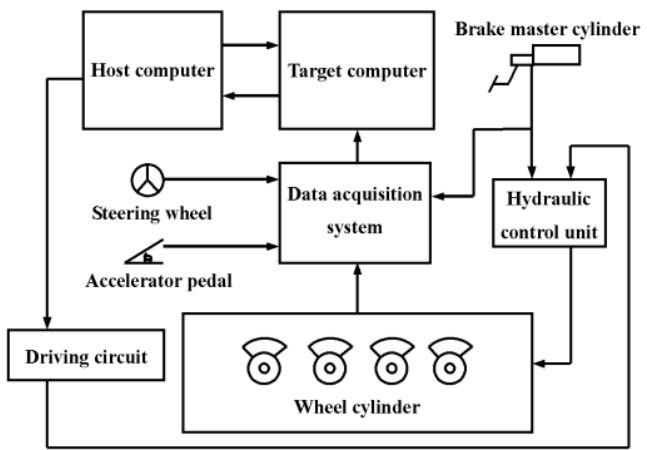

Figure 3. Electric-wheel vehicle ESC test platform sketch map

The host computer and the target computer were connected by the TCP/IP. The signals of the steering wheel, accelerator pedal, and brake master cylinder and wheel cylinder were transmitted to the data acquisition system by the CAN. The target computer received signals from the data acquisition system by the CAN and transferred them to the host computer by TCP/IP.

The experiments included a step input experiment in $70 \mathrm{~km} / \mathrm{h}$ and a step input experiment in $110 \mathrm{~km} / \mathrm{h}$. The purpose of the experiments was to verify the effectiveness of the improved soft measurement algorithm. This was used to estimate the yaw velocity and sides lip angle.
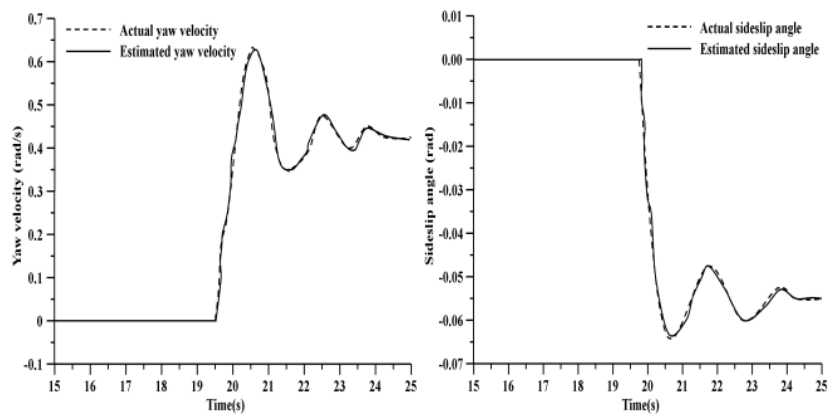

Figure 4. Step input experiment results in 70 kilometers per hour
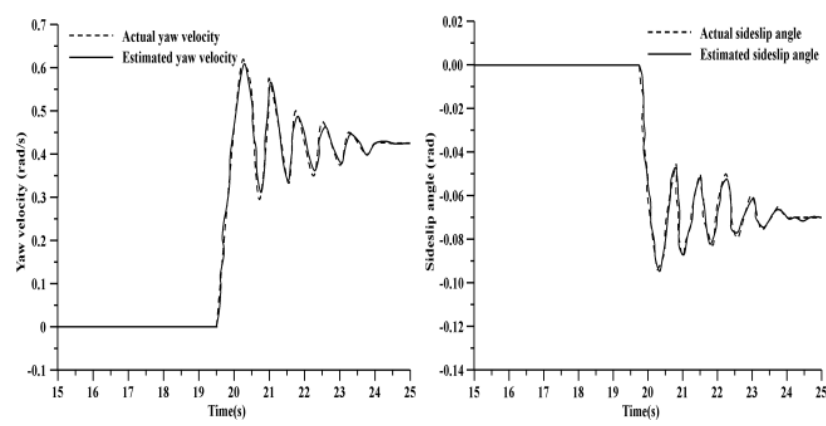

Figure 5. Step input experiment results in 110 kilometers per hour

The experimental results were shown as the Fig. 4 and the Fig. 5. From the experimental results, it could be seen that the estimated value of yaw rate and sideslip angle tracked the actual very well. The maximum absolute error of the estimated value and the actual value was small. The estimated values could be used to instead of the actual values for ESC. The overshoot of the tracking process was small and the lag time was short.

\section{CONCLUSIONS}

The three degree of freedom vehicle model is beneficial to simplify the modeling of the estimation for electric-wheel vehicle. The vehicle soft measurement model is fit for the soft measurement algorithm. The improved UKF sampling strategy can speed up the system sampling rate. The improved UKF algorith $m$ can enhance the accuracy of soft measurement. The estimated values of the yaw velocity and sideslip angle tracked the actual values very well adopting the improved UKF algorithm for ESC. The timeliness and accuracy of the soft measurement method of the ESC were enhanced.

\section{REFERENCES}

[1] He Ren, Zhang Ruijun, "Research and development of in-wheel motor drive technology," China Journal of Chongqing University of Technology, vol.29, pp. 10-13, July 2015.

[2] Chen Hui. Gao Bolin, Xu Fan, "Review on vehicle sideslip angle estimation," China Journal of Mechanical Engineering, vol. 49, pp. 77-80, December 2013.

[3] Zhang Xiaolong, Li Liang, and Song Jian, "Performance test and data processing method for vehicle electronic stability control system," Transactions of the Chinese Society for Agricultural, vol. 42, pp. 2-6, May 2011.

[4] Liang Songfeng, Qiu Hao, and Dong Zhurong, "3-DOF modeling and verification for electric vehicle based on vector space," China Journal of Control Engineering, vol. 22, pp. 79-84, January 2015.

[5] Yu Zhuoping, Gao Xiaojie, "Review of vehicle state estimation problem under driving situation," China Journal of Mechanical Engineering, vol. 45, pp. 20-28, May 2009.

[6] Liu Sheng, Zhang Hongmei, Optimal estimation theory, 1st ed., Science Press: Peking, 2011, pp.130-140.

[7] Lu Bing, Xie Xiaohua, and Cai Ketian, "Speed estimation research and simulation based on UKF algorithm," China Journal of Jilin University, vol. 33, pp. 8-11, January 2015.

[8] S. Jafarzadeh, C. Lascuc, and S. Fadali. M, "State estimation of induction motor drives using the unscented kalman filter", ndustrial Electronics, vol. 59, pp. 10-18, November 2012.

[9] Gao Lijun, Liu Qi, "Improved UKF algorithm based on cauchy robust function", China Journal of East China University Science and Technology, vol. 41, pp. 244-249, April2015.

[10] Lin Tao, Liu Yian, and H. Chao, "Application of improvement UKF algorithm in over the horizon radar", China Journal of Computer Simulition, Vol. 31,pp. 6-9, June 2014. 